\title{
Effects of US habituation following skin-conductance response conditioning: Support for a Pavlovian S-S position and a habituation account of nonmonotonic acquisition functions
}

\author{
CONSTANTINE X. POULOS \\ Addiction Research Foundation, Toronto, Canada \\ and \\ JOHN J. FUREDY and RONALD J. HESLEGRAVE \\ University of Toronto, Toronto, Ontario MSS 1A1, Canada
}

\begin{abstract}
After 48 subjects were given differential skin-conductance response (SCR) conditioning (six trials each with CS+ and CS-) with a 4-sec CS+US interval and a 100-dB white noise as US, they were divided into three equal groups in a treatment phase that comprised the following conditions for the three groups. For the conditioning group, there were 70 trials of the US paired with CS+ (i.e., CS+US conditioning trials) interspersed with 70 CStrials (i.e., unpaired CS trials for differential conditioning), at a mean intertrial interval of $20 \mathrm{sec}$; for the habituation group, the 70 noise USs were presented at the same time as those for the conditioning group, but no CSs were given; for the control group, the conditions of the habituation group were duplicated, except that another strong stimulus, a 1.5$\mathrm{mA}$ shock, replaced the noise US. Following the treatment phase, the effects of the different treatments on differential conditioning were assessed in a final test phase by presenting five CS+ and five CS- stimuli. The initial acquisition stage produced significant and equal SCR differentiation in all three groups, and all three treatments resulted in total habituation of the SCR to the aversive stimulus. Differential conditioning was totally absent during the test phase, not only in the conditioning but also in the habituation group; it was preserved, however, in the control group, which had been (totally) habituated to a stimulus modally different from the US. It is argued that these results support an S-S over an S-R interpretation of conditioning, and therefore provide an empirical extension of the pattern of results reported earlier in response-suppression paradigms wherein the mediational CRs are not directly observed. Also, the results suggest that US habituation is responsible for the nonmonotonic acquisition functions that are often seen in human differential SCR conditioning.
\end{abstract}

The initial impetus for the present study came from an experiment by Rescorla (1973) which had direct implications for S-S and S-R views of classical conditioning. That experiment used a conditionedsuppression procedure with rats and involved initial "fear" conditioning with a loud-noise unconditional stimulus (US) which was followed by habituation of that US. Subsequent testing showed that US habituation attenuated the previously established conditional response (CR). Since no extinction pro-

This research was supported by grants from the Canadian National and Medical Research Councils to J. J. F. We are indebted to H.D. Kimmel and W. F. Prokasy for critical comments on an earlier draft. Address reprint requests to John J. Furedy, Department of Psychology, University of Toronto, Toronto, Ontario M5S 1A1, Canada. cedures were involved in the US habituation phase which would affect the $S-R$ associative connection, the $S-R$ position would seem to have difficulty in accounting for the attenuating effect on the previously established CR. On the other hand, S-S accounts assume that the conditional stimulus (CS) elicits some representation of the US or, in Pavlovian terms, comes to activate the US center. Thus an S-S view readily accounts for a finding that degrading the US representation or center (e.g., by habituation) will degrade CRs based on that US. In short, the experiment provides a test for determining the implications of the S-S and S-R views of classical conditioning.

The present experiment employed similar experimental logic with humans in a differential skin- 
conductance response (SCR) conditioning paradigm in which the CRs were directly measured. Gormezano and Kehoe (1975) have elaborated in detail the need for the establishment of empirical convergence between the findings from "CS-IR" (e.g., conditioned suppression, CER procedures) and "CS-CR" (classical conditioning involving direct measurement of the target $\mathrm{CR}$ ) paradigms.

To investigate the validity of extending Rescorla's findings to CS-CR paradigms, three independent groups were run in a human differential SCR conditioning preparation. Initially, all groups received a series of differential conditioning trials with loud noise serving as the US. Following this acquisition training, the treatment phase consisted of the following arrangements: one group continued to receive further differential conditioning (conditioning group), a second group received loud-noise US-alone habituation trials (habituation group), and the final group received shock-alone habituation trials (control group). In the final test phase, conditioning was assessed for all groups by $\mathrm{CS}+$ and $\mathrm{CS}-$ alone trials to determine the degree of conditioning remaining after further conditioning, habituation to the noise US, or habituation to another modally different stimulus (i.e., shock) which was strong enough to serve as a US in other conditioning experiments. The S-S position would seem to predict that the conditioning and habituation groups would evidence diminished differential conditioning relative to the control group, since the noise US, and thus the CR, would have been degraded in both of these groups because of repeated exposures to the US. Within the context of the S-S prediction, it should be noted that the control group controls for such factors as fatigue, attention, and conditioning to background cues. Specifically, if the control group shows less reduction in differential conditioning than the habituation group, then it is the degradation of the US, or the representation of that US, that is shown to be critical. On the other hand, the S-R position would seem to predict that the degradation of the US would have no effect on the CR that develops during acquisition. Thus the habituation and control groups should show equivalent differential conditioning remaining in the test phase.

The present study also has direct implications for human SCR conditioning per se. A continuing apparent theoretical paradox in SCR conditioning is the frequent finding that acquisition training produces an inverted U-shaped function in the target CR (e.g., Grings, Lockhart, \& Dameron, 1963; Kimmel, 1959; Meryman, 1953). Various theoretical accounts have been suggested (cf. Kimmel, 1966; Kimmel \& Burns, 1975). However, if US habituation entails a decrement in the $\mathrm{CR}$, then a simple solution to the phenomenon becomes available. Events such as loud noise and shock which serve as USs in typical human SCR experiments are by almost any definition quite modest, and more importantly, are easily habituated. Thus, if habituation is occurring during the acquisition operations of conditioning, a nonmonotonic target CR function would no longer be viewed as paradoxical, but would be expected to occur.

\section{METHOD}

\section{Subjects and Apparatus}

The subjects were 48 undergraduates from the University of Toronto. Each subject was seated in a room separate from, but in continuous intercom contact with, the experimenter. The CSs in this experiment were two $28-\mathrm{V}$ lights, each of which illuminated either a red or green jewel lens. The US was a white-noise stimulus originating from a Grason-Stadler noise generator; it was delivered to the subject at $100 \mathrm{~dB}$ SPL as measured at the earphones. The shock stimulus was delivered through EEG electrodes filled with Grass EC-2 electrode cream, and the electrodes were placed $3 \mathrm{~cm}$ apart on the right upper arm. The shock originated from a constant-current source, and its intensity was set at $1.5 \mathrm{~mA}$.

The skin-resistance response (SRR) was measured by imposing a constant current of $70 \mu \mathrm{A}$ across zinc-manganese-dioxide electrodes, with a $5 \%$ saline solution as the conducting medium, which were placed on the left palm and right upper arm. This configuration minimizes the shock-SRR artifact where the shock is reported to be felt through the neutral (separately, but not floatingly, grounded) SRR electrode (Furedy, 1971, p. 498). The SRR was amplified by a Fels dermohmmeter and recorded by an Esterline-Angus rectigraph.

\section{Procedure}

Prior to commencing the initial acquisition phase, subjects randomly received two presentations of each of the 4-sec CSs in order to reduce any orienting responses to those visual stimuli.

The acquisition phase was identical for all groups with the CSs $(\mathrm{CS}+$ and $\mathrm{CS}-)$ balanced, using a Latin square procedure, for both color and position within each group of subjects. All groups received six CS + US trials and six CS - trials in a differential, delay-conditioning paradigm with the 4-sec CS + terminating at the onset of the $.3-\mathrm{sec}, 100-\mathrm{dB}$ white-noise US. The sequence of trials was randomly determined and the intertrial intervals (ITIs) were randomly varied among 15,20 , and $25 \mathrm{sec}$ with a mean ITI of $20 \mathrm{sec}$.

During the treatment phase, the three groups received different manipulations. The conditioning group merely received further differential conditioning which consisted of $70 \mathrm{CS}+\mathrm{US}$ and 70 CS - presentations. The CS + US and CS - temporal presentations were randomly determined, as in the acquisition phase, with a resulting mean ITI of $20 \mathrm{sec}$. The habituation group was habituated to the noise US by 70 US-alone presentations in which the inter-US-interval exactly mirrored the US presentations of the conditioning group. The control group was identical to the habituation group, with the exception that the noise US was replaced with a shock stimulus. More specifically, the control group received 70 shock-alone events. The inter-shock-interval mirrored the US presentations in the conditioning group.

The final test phase involved assessing the degree of conditioning remaining following further conditioning (conditioning group), habituation to the noise US (habituation group), or habituation to a different aversive shock stimulus (control group). All groups received a random presentation of five $\mathrm{CS}+$ alone, five $\mathrm{CS}-$, and five noise US-alone events. The inclusion of US-alone presentations was to mitigate rapid extinction of any remaining CRs. Half the subjects in each group received the $\mathrm{CS}+$ as the initial trial, while the remaining subjects received the CS - as the initial trial of this phase in an attempt to equate for orienting responses. 
Again the ITIs were randomly varied between 15, 20, and $25 \mathrm{sec}$, with a mean ITI of $20 \mathrm{sec}$.

\section{RESULTS}

The SRR was defined as a drop in resistance from 1 to $5 \mathrm{sec}$ following stimulus onset, and was transformed into SCR micromho units for the analysis.

The initial analysis was conducted on the acquisition phase to determine if differential conditioning had developed in the six conditioning trials. The ANOVA with Groups (i.e., conditioning, habituation and control), CS (i.e., CS + and CS -), and Trials as the factors yielded a significant $C S$ effect $[F(1 / 45)=$ $16.65, \mathrm{p}<.001]$, a significant Trials effect $[\mathrm{F}(5 / 225)$ $=3.42, \mathrm{p}<.01]$, and a significant $\mathrm{CS}$ by Trials interaction $[\mathrm{F} 5 / 255)=3.89, \mathrm{p}<.01]$, using a significance level of .05 for all comparisons. Figure 1 shows these results; as can be seen, the interaction is due to the development of differential SCR conditioning in the last four trials.

In order to determine the degree of conditioning achieved for each group in the acquisition phase and to check that the resulting conditioning was not biased in favor of either the S-S or S-R position, only the last four trials were analyzed. This analysis revealed that the SCRs to the CS + significantly exceeded those to the CS $-[F(1 / 45)=19.34, p<.001]$, and this differentiation was not differential between groups (i.e., the CS+/CS- by Groups interaction was not significant, $F<1$ ). The left panel of Figure 2 shows the resulting mean SCR difference $(C S+$ minus CS-) scores for the three groups during the last four trials of the acquisition phase.

An analysis of the treatment phase of the experiment was conducted to confirm that the experimental

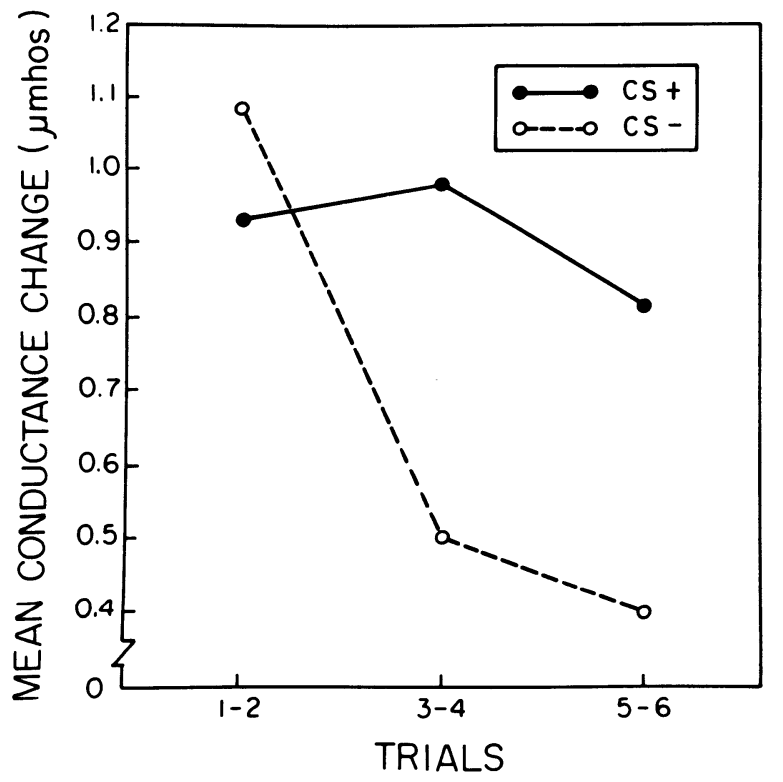

Figure 1. Mean SCRs to CS + and CS - trials during acquisition.

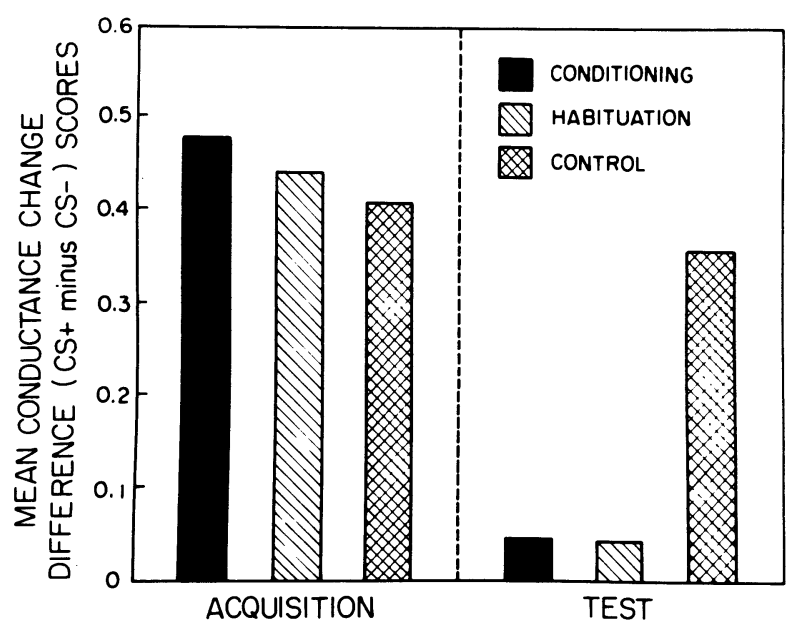

Figure 2. Differential SCR (micromhos) conditioning for all groups during the acquisition and test phases.

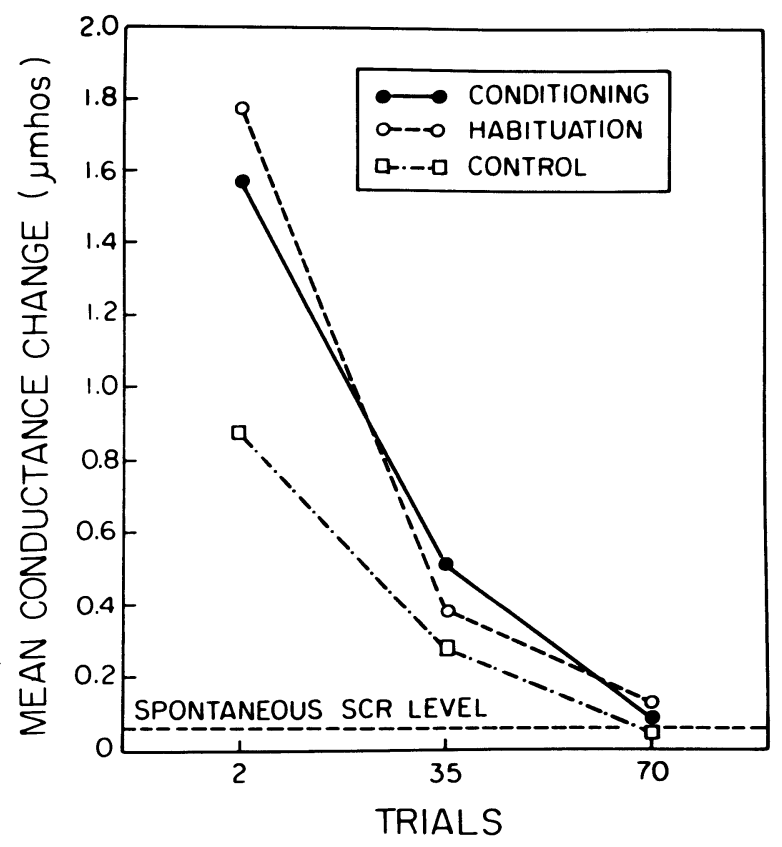

Figure 3. Mean noise US or shock-elicited SCRs for all groups during the treatment phase and the spontaneous SCR level prior to Trial 70.

manipulation of habituation to both the noise US and the shock had indeed resulted. Three trials, representing the beginning (No. 2), middle (No. 35) and end (No. 70) of this phase, were selected for each subject to estimate the degree of habituation across the entire phase; Trial 2 was selected instead of Trial 1 , however, to avoid unreliable responses which may have occurred on the first trial of this phase. ${ }^{1}$ Figure 3 shows the results of this analysis, and it is clear that significant habituation to both the noise US and shock occurred over this phase as indicated by the highly significant decline in the absolute level of SCR responding over trials $[\mathrm{F}(2 / 90)=68.12, \mathrm{p}<.001]$, 
asymptoting near zero by the final trial of the phase. There was also a significant Group by Trials interaction $[\mathrm{F}(4 / 90)=2.91, \mathrm{p}<.05]$, which was due to differences between groups at the beginning of this phase, as can be seen in the figure. Simple main effects revealed that groups differed only on Trial 2 $[F(1 / 45)=7.57, p<.01]$. Groups were not different at the middle or end of this phase, as indicated by the respective simple main effects determined (Fs $<1)$.

In order to determine whether the SCR to the noise US and the shock were totally habituated, spontaneous SCR levels were estimated for each subject by scoring the ITI from 9 to $5 \mathrm{sec}$ before the onset of Trial 70. This level of spontaneous SCRs for all groups ( $\overline{\mathrm{X}}=.058 \mu \mathrm{mhos})$ is shown in Figure 3. Statistical analysis indicated that, by Trial 70 , the difference between spontaneous and (stimulus) elicited SCR levels was not significant in either the two noiseUS groups or the shock group, which indicated that total habituation to the noise US and shock had resulted from the habituation treatment-phase procedure.

The right panel of Figure 2 shows the mean CS+ minus CS- SCR difference scores during the test phase which followed further conditioning, noise US habituation, or shock habituation for the three groups, respectively. The general analysis of this phase revealed that there were still significantly greater SCRs to the CS + than to the CS $-[F(1 / 45)=4.79$, $\mathrm{p}<.05$ ]. A subsequent analysis was conducted to determine the relevant simple main effects, i.e., the difference between the $\mathrm{CS}+$ and $\mathrm{CS}-$ for each group. As expected from the figure, only the shockhabituated control group showed significantly greater responding to the $C S+$ than to $C S-[F(1 / 45)=9.45$, $\mathrm{p}<.01]$; the other two groups showed only minimal and nonsignificant differentiation $(\mathrm{Fs}<1)$.

\section{DISCUSSION}

The pattern of results produced in this experiment is clear. ${ }^{2}$ The initial acquisition phase produced significant and comparable differential SCR conditioning in the three groups. The treatment phase, which involved repeated trial exposures, yielded significant and comparable response decrements to the noise US when that US was separately presented alone (habituation group) or was preceded by the CS + during further acquisition training (conditioning group). Responsivity to the shock was also significantly reduced by shock-alone presentations in the control group. Finally, the test phase revealed that differential conditioning survived only in the control group, which had not been exposed to the multiple repeated presentations of the noise US during the second phase either in the form of US-alone (habituation group) or CS + US noise (conditioning group) presen- tations. There are a number of implications of these findings.

First, the observation that conditioning survived for the control group but not for the habituation or conditioning groups provides evidence consistent with an S-S view of conditioning. The language of such S-S views varies. Pavlov (1927) spoke in terms of connections to US centers. Description in terms of memory have also been used (e.g., Rescorla, 1973; Wagner, 1976); that is, the CS is said to elicit some representation or memory of the US. However, the important conceptual feature is that if the US representation or center is reduced or degraded by any independent operation (in this experiment, by US repetition leading to habituation), there will be a corresponding decrement in conditional responsivity to the CS. An S-R position, of course, speaks in terms of response tendencies being directly associated with the CS such that any degrading of the US by independent operations would not be predicted to have an effect on the CR. The present findings in this regard were particularly clear. Habituation of the noise US completely eliminated the previously established conditioning, whereas the condition which had been exposed only to shock-alone presentations (i.e., control group) maintained the previously established conditioning. It bears noting that the effects of repeated exposure were specific and cannot easily be accounted for in terms of some general receptor fatigue notion, or in terms of the conditioning of aversiveness to situational cues, since the control group received shockalone presentations during the treatment phase. The specificity of the effects of US exposure provides added evidence for an S-S view in terms of which any degrading of another (here shock) US center or representation would not affect responsivity associated with the target US (here noise). The present findings are also of interest because they provide an instance of empirical convergence between indirect measures of classical conditioning (i.e., conditioned suppression procedures, Rescorla, 1973) and directly measured CRs (cf. Gormezano \& Kehoe, 1975).

The results also seem to provide strong support for the position that US habituation is the key factor in nonmonotonic SCR acquisition functions. The similar habituation patterns in the habituation and conditioning groups and the finding that US-alone habituation was functionally equivalent to additional $\mathrm{CS}+\mathrm{US}$ trials in producing decreased responding would seem to be strong support for the view that it is habituation to the US which is the critical factor in nonmonotonic acquisition functions. It bears noting that if US habituation occurs over conditioning trials, then the $S-R$ position can also account for nonmonotonic CR functions. The operative factor in the $S-R$ view would be that an ineffective (non-responseproducing US) would operationally define extinction, 
hence lead to the same prediction for the conditioning groups as the S-S prediction. Thus, from either an S-S or an S-R view, the nonmonotonic functions of SCR conditioning presumably reflect the high habituability of the US generally employed in human SCR studies. Moreover, any factors which would operate to reduce habituation to the US (e.g., longer ITIs and variation of the USs, etc.) should operate to reduce the response decrement during SCR acquisition training.

Finally, it should be noted that the present findings raise some interesting questions with respect to the process of habituation. Recently, Wagner (1976) proposed an information processing model for habituation. In support of this model, Wagner, Whitlow, and Pfautz (cited in Wagner, 1976) demonstrated that a group of rabbits that received additional exposure to the environment in which vasomotor habituation to an auditory stimulus had previously occurred showed significantly greater recovery of the habituated response relative to the control group without the additional exposure. In short, it was shown that situational cues are (associatively) critical for habituation. Such a factor could have been operating in the present experiment. However, it would also be expected that the conditioning group should habituate much more rapidly than the habituation group, since there were specific temporal cues (i.e., the $\mathrm{CS}+$ ) for the conditioning group and no such temporal cues for the habituation group during the treatment phase. In Wagner's model, a theoretical mechanism is provided whereby predictive cues result in reduced responsivity (i.e., habituation). It is surprising, based on Wagner's model, to find that the rate of habituation for the conditioning and habituation groups did not differ. The question arises as to why the conditioning group, which had the most informative cues (i.e., the CS +), does not produce habituation rapidly or at least more rapidly than the habituation group. In other words, it would seem that, at the very least, one must assume that separate associative factors are involved in conditioning and habituation. The question whether these separate factors are in terms of cues or processes remains, at this stage, a very open one.

\section{REFERENCES}

Furedy, J. J. Explicitly-unpaired and truly-random CS - controls in human classical differential autonomic conditioning. Psychophysiology, 1971, 8, 497-503.

Gormezano, I., \& Kеноe, E. J. Classical conditioning: Some methodological-conceptual issues. In W. K. Estes (Ed.), Handbook of learning and cognitive processes ( $\mathrm{Vol} .2)$ Conditioning and behavior theory. Hillsdale, NJ: Erlbaum, 1975.

Grings, W. W., Lockhart, R. A., \& Dameron, L. E. Conditioning autonomic responses of mentally subnormal individuals. Psychological Monographs, 1963, 76(39, Whole No. 558).

KIMMEL, H. D. Amount of conditioning and intensity of conditioned stimulus. Journal of Experimental Psychology, $1959, \mathbf{5 8}, 283-288$.
Kimmel, H. D. Inhibition of the unconditioned response in classical conditioning. Psychological Review, 1966, 73, 232-240.

Kimmel, H. D., \& Burns, R. A. Adaptational aspects of conditioning. In W. K. Estes (Ed.), Handbook of learning and cognitive processes (Vol. 2) Conditioning and behavior theory. Hillsdale, N.J.: Erlbaum, 1975.

Meryman, J. J. The magnitude of an unconditioned GSR as a function of fear conditioned at a long CS-UCS interval. Unpublished PhD dissertation, University of Iowa, 1953.

Pavlov, I. P. Conditioned reflexes. London: Oxford University Press, 1927.

RESCORLA, R. A. Effect of US habituation following conditioning. Journal of Comparative and Physiological Psychology, 1973, 82, 137-143.

WAgner, A. R. Priming in STM: An information-processing mechanism for self-generated or retrieval-generated depression in performance. In T. J. Tighe \& R. N. Leaton (Eds.), Habituation: Perspectives from child development, animal behavior, and neurophysiology. Hillsdale, N.J: Erlbaum, 1976.

\section{NOTES}

1. The control group subjects would be especially likely to produce unreliable responses due to movement artifacts since the first trial of the treatment phase was their initial experience with the shock.

2. H. D. Kimmel has suggested a potential source of confounding that could have made the interpretation of the results more difficult, but the actual results indicate that this source of confounding was not, in fact, present in the experiment. Kimmel's suggestion is that the inclusion of noise-alone presentations during the testing phase (to reduce the rate of extinction) could have biased the results in favor of the control group, since, for this group, after the 70 -shock presentations in the second phase, the reintroduction of the noise during the test phase could have led to dishabituation or a general elevation of response levels. Such a dishabituation effect would increase responding to both the CS + and $\mathrm{CS}-$ in the control group and thereby lead to the differentiation being more "visible" in this group. However, the absolute response levels elicited by the CSs during the test phase in the three groups do not support the dishabituation interpretation. Mean CS + responding for control, habituation, and conditioning groups, respectively, were $.587, .254$, and .189 , while the corresponding CS - values were $.224, .210$, and .143. A Tukey test of multiple comparisons indicated that the CS + value of the control group was significantly greater than the other, statistically homogeneous CS values, whereas Kimmel's suggestion would seem to minimally require that the CS - level of the control group would also be elevated (through dishabituation).

As noted by a consultant, it is also possible to raise a "selective" version of the dishabituation argument according to which the reintroduction of the noise in the control group dishabituates only the "associative response" to CS + and not that to $\mathrm{CS}-$. However, the results do not support the view that such a selective (associative) sort of dishabituation was operating. The data most relevant to this issue are those based on the first (before the introduction of the noise) and second (after the introduction of the noise) set of CS + and CS - trials in the test phase of the control group. Orthogonal polynomials showed only a significant CS effect $[F(1 / 45)=11.05, p<.01]$, with $C S+$ performance exceeding $\mathrm{CS}-$ performance, but no $\mathrm{CS}$ by trials interaction $(F<1)$ of the sort required by the selective dishabituation account, according to which the CS effect should have emerged only on the second (after noise introduction) set of CS trials.

(Received for publication October 27, 1978; revision accepted July $17,1979$. ) 